\title{
A retrospective analysis on the effect of single-channel minimally invasive percutaneous nephrolithotomy combined with retrograde flexible ureteroscopy using the completely lateral decubitus and semi-lithotomy positions to treat complex kidney stones
}

\author{
Dongliang Pan ${ }^{1}$, Lufang Zhang ${ }^{2}$, Jiaxu Pan $^{3}$, Bing Yang ${ }^{1}$, Pengfei Gao ${ }^{1}$, Keping Zhang ${ }^{2}$ \\ ${ }^{1}$ Department of Urologic Surgery, Peking University Shougang Hospital, Beijing, China; ${ }^{2}$ Department of Urologic Surgery, Weifang People's \\ Hospital, Weifang, China; ${ }^{3}$ Division of management, School of Business, Massey University, Auckland, New Zealand \\ Contributions: (I) Conception and design: D Pan, L Zhang; (II) Administrative support: D Pan; (III) Provision of study materials or patients: D Pan, \\ L Zhang; (IV) Collection and assembly of data: B Yang, P Gao, K Zhang; (V) Data analysis and interpretation: J Pan; (VI) Manuscript writing: All \\ authors; (VII) Final approval of manuscript: All authors. \\ Correspondence to: Lufang Zhang. Department of Urologic Surgery, Weifang People’s Hospital, Weifang 261041, China. Email: zhanglufang@hotmail.com.
}

Background: Some types of complex kidney stones cannot be broken down and removed through singlechannel percutaneous nephroscope or retrograde flexible ureteroscope. In order to be removed, these types of stones require multiple combined methods to be performed. The aim of this study was to retrospectively evaluate the clinical effect of single-channel minimally invasive percutaneous nephrolithotomy (mPCNL) combined with retrograde flexible ureteroscopy using the completely lateral decubitus and semi-lithotomy positions for treating complex renal calculi.

Methods: We selected 117 patients with complex renal calculi who were admitted to Peking University Shougang Hospital and Weifang People's Hospital from January 1, 2017, to January 31, 2021. All patients were treated with single-channel mPCNL combined with retrograde flexible ureteroscopy in the completely lateral decubitus and semi-lithotomy positions. During the operation, the patients were placed in a completely lateral decubitus position, or their lower limbs were placed in a semi-lithotomy position for a single attempt only.

Results: An 18-Fr percutaneous channel was successfully established in all patients. The mean operation time was $112 \pm 37$ minutes, and the average blood loss was $71 \pm 31 \mathrm{~mL}$. A 14-Fr renal fistula was maintained for 7 days, a urethral catheter for 2-3 days, and a ureteral stent tube for 2 weeks after each surgery. According to the results of computed tomography (CT) scans performed 3-5 days after the operation, the total lithotripsy success rate reached $100 \%$, with a first-stage lithotripsy rate of $98.29 \%$. Two patients were found to each have 1 residual stone, with a diameter of $4 \mathrm{~mm}$, left in kidney by CT, which then was to be removed under local anesthesia. The average postoperative hospitalized time was $7 \pm 2$ days, and no severe complications occurred perioperatively.

Conclusions: Single-channel mPCNL combined with retrograde flexible ureteroscopy in the completely lateral decubitus and semi-lithotomy positions is a safe, feasible, and highly effective method of treating complex renal calculi, which is of benefit to save operation time and facilitate operation process, because patient's position could not need to be changed repeatedly during the surgery.

Keywords: Completely lateral position; semi-lithotomy position; minimally invasive percutaneous nephroscopy; flexible ureteroscope; complex kidney stones

Submitted Jun 29, 2021. Accepted for publication Aug 12, 2021.

doi: $10.21037 / \mathrm{tau}-21-635$

View this article at: https://dx.doi.org/10.21037/tau-21-635 


\section{Introduction}

Complicated kidney stones, such as staghorn kidney stones, multiple kidney stones, and single kidney stones with a diameter $>2.0 \mathrm{~cm}$, are difficult to treat $(1,2)$. At present, minimally invasive percutaneous nephrolithotomy (mPCNL) has become an effective method for the clinical treatment of upper urinary stones (3-6). Some types of complex kidney stones cannot be broken down and removed through single-channel mPCNL. These complex kidney stones include those outside the primary grouping of kidney stones that are parallel to the skin-kidney puncture channel, stones with an infundibulopelvic angle (IPA) angle of less than $80^{\circ}$, and stones with diverticula that cannot be reached by a percutaneous nephroscope. In order to be removed, these types of stones require multiple skinkidney puncture channels, puncture needle movement, a flexible nephroscope combined with a retrograde flexible ureteroscope, a flexible cystoscope, or even choledochoscopy to be performed (7-12). However, the creation of multiple percutaneous renal puncture channels may increase the damage to the kidney parenchyma and negatively impact renal function. Furthermore, the puncture needle movement technique is less effective for calculi in calyces or diverticula with a narrow neck due to the bending radius of flexible nephroscope. Furthermore, a flexible cystoscope and a choledochoscope are sometimes restricted by the space in the renal pelvis. Therefore, mPCNL combined with retrograde flexible ureteroscopy can overcome these technical shortcomings and increase the success rate of $\mathrm{mPCNL}$ lithotripsy.

In recent years, the surgical positioning of patients receiving $\mathrm{mPCNL}$ combined with retrograde flexible ureteroscope during lithotripsy has changed from alternating between the prone position and the lithotomy position $(10,13-16)$ to a one-time oblique supine lithotomy position (17-21). Maintaining a single position can reduce the negative impact to the patient's cardiopulmonary function, minimize interference to the operation, and decrease the physical exertion of medical staff. In order to improve the convenience of the procedure and the lithotripsy rate, from January 1, 2017, to January 31, 2021, Peking University Shougang Hospital and Weifang People's Hospital in Shandong Province both adopted singlechannel minimally invasive nephrolithotomy combined with retrograde flexible ureteroscopic holmium laser lithotripsy using the completely lateral decubitus and semi-lithotomy positions for the treatment of 117 patients with complicated kidney stones, the results of which were satisfactory. We present the following article in accordance with the STROBE reporting checklist (available at https://dx.doi. org/10.21037/tau-21-635).

\section{Methods}

\section{General information}

For this study, 117 patients with complicated kidney stones admitted to Peking University Shougang Hospital and Weifang People's Hospital in Shandong Province from January 1, 2017, to January 31, 2021 were enrolled (as shown in Table 1). All procedures performed in this study involving human participants were in accordance with the Declaration of Helsinki (as revised in 2013). The study was approved by the Ethics Committee of Peking University Shougang Hospital (No. IRB-SOP-21-00) and informed consent was taken from all the patients. All had large pelvicalyceal calculi [diameter $2.1-5.3 \mathrm{~cm}(3.53 \pm 2.38 \mathrm{~cm})$ ] which a single skin-kidney puncture channel could not completely break down. Of these 117 patients, 59 had calculi parallel to the skin-kidney puncture channel in addition to primary renal calculi, 41 cases had calyceal calculi with an IPA less than $80^{\circ}$, and 17 cases had calculi in renal calyces that a percutaneous nephroscope could not reach. There were 96 males and 21 females. Patient age ranged from 23 to 77 years old, giving an average of 41.4 years. The body mass index range was $21-43$ $\left(27.41 \pm 8.19 \mathrm{~kg} / \mathrm{m}^{2}\right)$. The reasons for the patients undergoing treatment were as follows: 14 cases of waist discomfort, 40 cases of hematuria under observed via microscopy, 37 cases of repeated urinary tract infection, 19 cases of urinary tract infection with gross hematuria, and 7 cases of stones found by ultrasound. All patients underwent urinary routine testing, urine culture, ultrasound, plain abdominal X-ray plain, and a urinary tract computed tomography (CT) scan with enhanced examination. Urine routine testing showed white blood cell levels between 5-207/HPF (high power field), 31 patients were shown to have Escherichia coli via urine culture, patient hemoglobin levels were $102-170 \mathrm{~g} / \mathrm{L}$, hematocrit levels were $30-47 \%$, blood uric acid levels were 331-602 $\mu \mathrm{mol} / \mathrm{L}$, and 17 patients showed blood creatinine levels of 133-156 $\mathrm{mol} / \mathrm{L}$. All included patients had normal parathyroid hormone levels. CT of the patient urinary systems showed 27 patients with no hydronephrosis, 34 patients with mild hydronephrosis, 53 patients with moderate hydronephrosis, and 3 patients 
Table 1 Patient characteristics and operative outcomes

\begin{tabular}{|c|c|}
\hline Parameter & Median (range) or $\mathrm{n}(\%)$ \\
\hline Total number & 117 \\
\hline Male & 96 \\
\hline Female & 21 \\
\hline Age & $41.4[23-71]$ \\
\hline BMI $\left(\mathrm{kg} / \mathrm{m}^{2}\right)$ & $27.41 \pm 8.19[21-43]$ \\
\hline Stone diameter $(\mathrm{cm})$ & $3.53 \pm 2.38[2.1-5.3]$ \\
\hline \multicolumn{2}{|l|}{ Previous lithotripsy operations } \\
\hline ESWL & $76(64.9)$ \\
\hline FURL & $21(17.9)$ \\
\hline PCNL & $13(11.1)$ \\
\hline Preoperative $\mathrm{Hb}(\mathrm{g} / \mathrm{L})$ & $130.2 \pm 22.5[102-170]$ \\
\hline \multicolumn{2}{|l|}{ Comorbid hydronephrosis } \\
\hline No & $27(23.1)$ \\
\hline Mild & $34(29.1)$ \\
\hline Moderate & $53(45.3)$ \\
\hline Severe & $3(2.6)$ \\
\hline Operation time (min) & $112 \pm 37[86-237]$ \\
\hline Estimated blood loss (mL) & $71 \pm 31[30-120]$ \\
\hline Transfusion needed $(n)$ & 0 \\
\hline Postoperative Hb (g/L) & $125.1 \pm 27.5[98-156]$ \\
\hline \multicolumn{2}{|l|}{ Postoperative complication } \\
\hline Fever & $7(5.9)$ \\
\hline Hematuria & $117(100.0)$ \\
\hline Perirenal exudate & $12(10.2)$ \\
\hline \multicolumn{2}{|l|}{ Analgesics intake } \\
\hline Duration of nephrostomy drainage (day) & $6 \pm 1[5-7]$ \\
\hline Hospital stay after surgery (day) & $7 \pm 2[5-10]$ \\
\hline Lithotripsy success rate & $117(100.0)$ \\
\hline One-stage stone clearance rate & $115(98.29)$ \\
\hline Second-stage lithotripsy rate & $2(1.71)$ \\
\hline
\end{tabular}

ESWL, extracorporeal shock wave lithotripsy; FURL, flexible ureteroscopic lithotripsy; PCNL, percutaneous nephrolithotomy.

with severe hydronephrosis. Furthermore, 76 patients were treated with extracorporeal shock wave lithotripsy and drug excretion, but the effects were unsatisfactory. Previously, 21 patients had undergone retrograde soft ureteroscopic

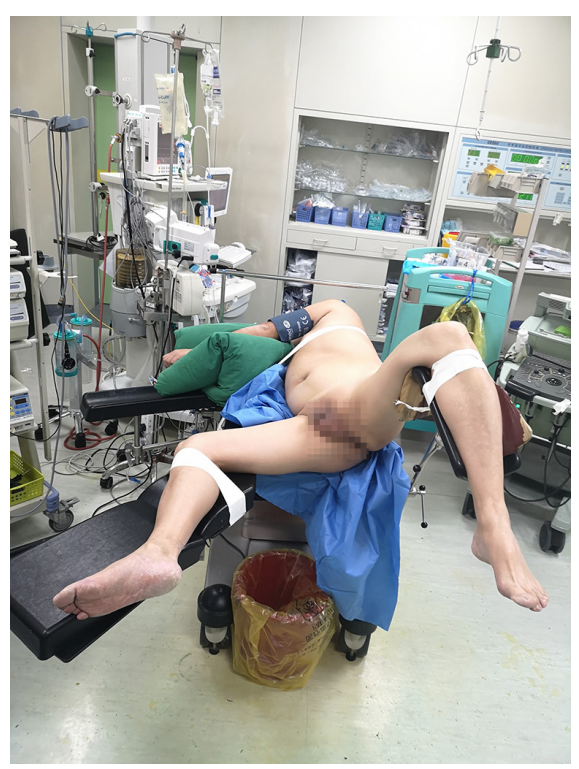

Figure 1 Intraoperative position for lithotripsy procedure in the complete lateral position.

lithotripsy through the urethra, and 13 patients had undergone a percutaneous nephrolithotomy procedure. All patients included in this study had recurrence of symptoms due to the residual stones. There were no contraindications for percutaneous nephroscopy noted for any of the patients.

\section{Treatment method}

\section{Preoperative preparation}

All patients signed an informed consent form for the operation. An intravenous cefuroxime injection was given to patients with a urinary tract infection 5-7 days before the operation until the urine routine test levels returned to normal. Patients with hypertension and diabetes had their blood glucose and blood pressure checked before the operation, and these were maintained within normal ranges.

\section{Surgical method}

General intravenous anesthesia was used for each procedure. The surgical position employed during the operations is shown in Figure 1. The patient would lie on their healthy side, exposing the abdomen and kidney area, and the backplane of the operating table was raised to about $90^{\circ}$ and secured with wide tape. The patient would then bend the lower extremity of their healthy side to about $150^{\circ}$ in a horizontal position, placing the thigh as close to the lower abdomen as possible. The lower limb of the affected side 
was flexed to about $90^{\circ}$ and abducted as much as possible; the patient's external urethral meatus was kept outside the lower edge of the operating bed to fully expose the patient's perineum. After disinfection, sterile towels were placed around the perineum and back surgical areas. An 8/9.6 Fr rigid ureteroscope was inserted into the bladder to find the ureteral orifice on the affected side, and a nickel-titanium guidewire was inserted into the ureteral orifice. Using a nickel-titanium guidewire, we entered the ureter through the rigid ureteroscope for inspection and then exited it. Assisted by the nickel-titanium guidewire, an 11/13 Fr (36 cm long) ureteroscope sheath was inserted into the ureter, and water was artificially injected into the renal pelvis through a flexible ureteroscope to cause hydronephrosis. The selected puncture point was the 11th or 12th subcostal region of the affected side, between the subscapular line and the posterior axillary line, located under ultrasound guidance. We punctured with an $18-\mathrm{Fr}$ Urovision puncture needle or Cliny puncture set for percutaneous nephrostomy, until fluid was observed to overflow from the puncture needle sheath. After confirming that fluid had entered the collection system, the safety guidewire was inserted, and the renal fistula mouth was dilated to 18 Fr starting from $10 \mathrm{Fr}$ using a fascial dilator, which established a percutaneous nephrolithotomy channel. An 8/9.6 Fr rigid ureteroscope was used to enter the collection system along the safety guidewire, and the surgical field was kept clear by flushing the perfusion pump to break down stones with a 365-micron holmium laser fiber set to 30-45 W (energy $1.5 \mathrm{~J}$, frequency $20-30 \mathrm{~Hz}$ ). After the stones had been broken down, they were washed out via the peeling sheath, and the renal pelvis and calyx were then checked to confirm the surgery's success. When no residual stones were observed by the rigid ureteroscope, the renal pelvis and renal calyx were examined by retrograde flexible ureteroscopy to crush and flush out any residual stones via the peeling sheath. After microchannel percutaneous renal ureteroscopy and retrograde flexible ureteroscopy confirmed that no residual stones were remaining in the renal pelvis or renal calyx, a ureteral stent and a $14-\mathrm{Fr}$ nephrostomy drainage tube were inserted into the ureter. The skin incision was then sutured, and the nephrostomy draining tube was fixed to the patient.

Hematuria was closely observed after the operation, and routine blood tests were checked regularly to record any changes to the hematocrit. On days 3 to 5 after the operation, a plain scan CT was performed to check for any residual stones.

\section{Observation indicators}

The operations were timed from the beginning of the retrograde ureteroscopy to the suturing of the skin. Intraoperative blood loss was calculated as follows: (preoperative hematocrit-postoperative hematocrit)/ preoperative hematocrit $\times$ body weight $\times 7 \%$. The standard for successful indwelling urinary catheter removal was the presence of a clear urine color without secondary infection and the ability for patients to walk after bed rest, while the standard for having no stone residue was for patients to have no stones present or to have the largest diameter of residual stone fragments be $\leq 4 \mathrm{~mm}$, as presented by plain CT. Preoperative and postoperative blood creatinine levels, the extravasation of perirenal perfusion fluid by ultrasound after surgery, complication rates, postoperative analgesic demands, postoperative hospitalization times, and stone removal rates were also recorded.

\section{Statistical analysis}

We used SPSS 13.0 software (IBM Corporation, Armonk, NY, USA) to perform a $t$ test or a chi-squared test. The significance level of the hypothesis test was $\alpha=0.05$.

\section{Results}

An 18-Fr percutaneous renal channel was successfully established in all 117 cases, and all were single channels. As shown in Table 1, the average operation time was $112 \pm 37 \mathrm{~min}$, and the mean blood loss was $71 \pm 31 \mathrm{~mL}$. There were no complications during the operations. Ultrasound results showed a postoperative incidence of perirenal perfusion extravasation of $10.2 \%(12 / 117)$. There were no changes to patient hemoglobin or blood creatinine levels before or after the surgeries, and no blood transfusions were needed. On average, the $14 \mathrm{Fr}$ nephrostomy tube was retained for 7 days, the urinary tube for 2 to 3 days, and the ureteral stent tube for 2 weeks. There were 7 patients with postoperative fever (body temperature $<39^{\circ} \mathrm{C}$ ). Each patient had gross hematuria to varying degrees after surgery, which disappeared after 2 to 5 days. Routine intravenous antiinfection treatment was performed for 2 days after each operation, and no analgesic drugs were used. Reexamination of the plain CT scans 3 to 5 days after the operations showed a lithotripsy success rate of $100 \%$, with a lithotripsy rate in the first stage of $98.29 \%$ (115/117). Additionally, 2 patients were found to each have 1 residual stone with a 
diameter of about $4 \mathrm{~mm}$ in the kidney, which were located at the internal orifice of the renal channel and in the renal pelvis. These residual stones were both removed under local anesthetic. The mean postoperative hospitalization time was $7 \pm 2$ days, and no serious complications occurred. After being discharged from hospital, all patients were followed up for 3 months, and no delayed bleeding or infection was observed.

\section{Discussion}

Single-channel mPCNL combined with retrograde flexible ureteroscope using the completely lateral decubitus and semi-lithotomy positions is a safe, effective, and feasible treatment for complex kidney stones. For cases of complex kidney stones, it is necessary to establish multiple channels at the same time due to difficulties in reaching the target calculi or diverticula through the single channel provided by traditional percutaneous nephroscopy. This traditional approach inevitably increases renal parenchymal damage and the risk of accidental injury to the surrounding organs and surgical bleeding. A solution to this issue was sought by either adding a soft ureteroscopy sheath to the lithotomy position to facilitate retrograde soft ureteroscopy lithotripsy or by increasing the model of the percutaneous renal sheath to try soft cystoscopy lithotripsy or flexible nephrolithotomy. However, since parts of the calyces or diverticula are too far away from the working channel of the skin-kidney, or the mouths of the calyx diverticula are too small, these solutions generally fail, with the number of residual stones being increased. Therefore, developing a method that can remove all complicated kidney stones with minimal damage has been the goal for urologists to explore and pursue. In recent years, based on the percutaneous minimally invasive single-channel approach, various hospitals have explored surgical methods for treating complex kidney stones with a single operation that causes little damage, but this approach has resulted in high lithotripsy rates. The one-stage single-channel puncture operation as described in this article avoids the excessive damage to the kidney parenchyma caused by multiple channels. Furthermore, its combination with retrograde flexible ureteroscopy assists in the positioning and visual expansion of the kidney puncture channel, reducing the risk of accidental puncturing of the renal column and subsequent tearing of the branch of the renal artery, and limits the swing amplitude of the rigid ureternephroscope during the operation. This approach also reduces the risk of bleeding caused by tearing of the renal parenchyma during the operation and increases the safety of the operation. The average blood loss during operation in the study group was $71 \pm 31 \mathrm{~mL}$. There were no changes in hemoglobin or blood creatinine levels before or after the operation and no reported blood transfusions. Blood loss was much lower than the reduction of hemoglobin $(10.5 \pm 3.3 \mathrm{~g} / \mathrm{L})$ after percutaneous nephrolithotomy (PCNL) reported in the literature (2) $[16.4 \pm 4.6 \mathrm{~g} / \mathrm{L}(22)$; 24.8-28 g/L (7)], which confirmed this method's safety. Single-channel minimally invasive PCNL combined with retrograde transurethral soft ureteroscopy for the treatment of complex kidney stones has been used in some hospitals (13-16). Compared to those provided by simple singlechannel minimally invasive PCNL, the average operation time and hospitalization time of single-channel mPCNL combined with retrograde flexible ureteroscopy were shorter and were accompanied by less bleeding and lower rates of complication; however, this success might have been due to the change in the position during the operation or reoperation. The double-lens combined lithotripsy operation performed in the oblique supine lithotomy position can avoid the extracorporeal transformation during the operation. However, since the oblique supine is usually $45^{\circ}$, the lithotripsy cannot be completely gathered in one place by gravity, and with the perfusion fluid drifting, the crushed stones need to be searched for repeatedly. We then attempted using a single-channel minimally invasive percutaneous nephroscope combined with retrograde reflexible ureteroscopic lithotripsy in completely lateral and semi-lithotomy positions, realizing that these positions can meet the needs of dual-path double-lens lithotripsy for surgery, with mPCNL being the main device used to perform the lithotripsy. The use of the retrograde soft ureteroscope was auxiliary, but its role was indispensable. The retrograde soft ureteroscope was the first device to enter the renal pelvis at the beginning of the operation, and it assisted in visually determining the percutaneous renal puncture point. When the nephroscope was used, it was retreated into the sheath of the flexible ureteroscope, while the retrograde flexible ureteroscope was only responsible for breaking down the stones that could not be reached by the percutaneous nephroscope in the renal pelvis. The broken down stones were passed to the percutaneous nephroscope, and as the single-channel percutaneous nephroscope did not need to swing excessively during the operation, the risk of tearing the renal parenchyma was reduced. The combination of using these two devices was 
shown to achieve twice the results with half the effort, saving on operation times and improving both lithotripsy rates and surgical efficiency. Therefore, the single-channel minimally invasive percutaneous nephroscope combined with retrograde soft ureteroscopy using the completely lateral and semi-lithotomy positions for the treatment of complex kidney stones has high safety, high efficiency, and good maneuverability.

In the treatment of complicated kidney stones with single-channel minimally invasive percutaneous nephroscope combined with retrograde soft ureteroscopy using the completely lateral and semi-lithotomy positions, the completely lateral position was shown to be essential for improving the removal rate of the stones. This is due to the patient being in a full lateral position, the renal pelvis outlet being at the lowest position, and almost all the calyces and diverticula being higher than the renal pelvis. Therefore, the fragments of broken stones would gradually and automatically collect into the renal pelvic and ureter junction under their own gravity during lithotripsy, provided that the maximum diameter of the deteriorated stones was smaller than the width of the inner diameter of the mouth of the diverticulum. We have employed various patient positions in combination with $\mathrm{MPCNL}$ to remove broken down stones. In the fully prone position, the fragments of broken stones would scatter throughout the multiple low positions of the renal pelvis and renal calyces due to the flow of perfusion water. The nephroscope was frequently needed to check and remove these stones, and a stone residue rate reaching $10-20 \%$ was present. Next, the $45^{\circ}$ oblique supine position helped the fragments of broken stones to converge in the renal pelvic ureteral junction, with the self-gravity of stone fragments; however, the openings of some individual posterior calyces might still have been in the highest position, hindering the movement of the gravel to the junction of the renal pelvis and ureter. When the patient was placed in the $75^{\circ}$ oblique supine position or the completely lateral position, the orifices of almost all calyx diverticula were lower than the lowest position of the renal calyces and diverticula. When the calyx diverticulum orifice was at the lowest position of the renal calyx and diverticulum, the working efficiency of retrograde soft ureteroscopic lithotripsy was higher, as the smaller gravel and powder would leave the renal calyx and diverticulum via gravity during the lithotripsy, with fewer and fewer residual stones in the renal pelvis and diverticulum remaining, indicating that more space had been created. When the lithotripsy was completed, there was often no gravel found in the renal calyx or diverticulum, which obviated the need for a stone basket, especially when the fully lateral position was employed. Meanwhile, when performing lithotripsy, small gravel and powder continuously left the renal calyces and diverticula, ensuring a clear field of view. Thus, retrograde soft ureteroscopy facilitates the continuation of the lithotripsy procedure and shortens the overall operation time. In this way, the completely lateral position can improve the lithotripsy rate.

Single-channel minimally invasive percutaneous nephroscopy combined with retrograde flexible ureteroscopy in the completely lateral and semi-lithotomy positions did not require patient repositioning during the complex kidney stone operations, which reduced the physical exertion of medical staff. Apart from the oblique supine position below $45^{\circ}$ combined with double scopes being used to perform lithotripsy, the complete prone position and the $75^{\circ}$ oblique supine position are usually adopted to insert a soft ureteroscope sheath under the patient in the lithotomy position and then to perform mPCNL, which requires medical workers to change the patient's position twice. In particular, the full prone position increases the physical exertion of medical workers and the amount of disinfection materials. Another negative impact of the prone position is that the cardiopulmonary function of some patients is greatly restricted, while the use of the completely lateral and semi-lithotomy positions were not shown to impact cardiopulmonary function. Furthermore, the benefit of using a completely lateral position and a semilithotomy position is that a patient needs positioning only at beginning of the operation, and the auxiliary materials for the upper and lower operation areas can be draped in place at the one time. In addition, compared to the complete lithotomy position, although the opening angle of the lower limbs is smaller in the semi-lithotomy position, the swing space available for rigid ureteroscopes in the complete lithotomy position is still sufficient. The upright position affects the operation of the ureteroscope, so the operating bed needs to be elevated, or the operator should sit on a low stool to adapt to the change of usual operating posture due to the patient's semi-lithotomy position.

The single-channel minimally invasive percutaneous nephroscope combined with retrograde soft ureteroscopy using the completely lateral and semi-lithotomy positions can also reduce the overall cost of treatment. Residual stones present after a single PCNL procedure usually need a second-stage operation for them to be completely removed. The second-stage operation still needs to be performed in 
an operating room, and the level and cost of the operation are essentially the same as those of the first-stage operation. The interval between the two operations is about 1 week. The second-stage operation increases a patient's medical expenses and the cost of family members' time. Retrograde ureteroscopic lithotripsy generally requires the use of a disposable stone retrieval basket for stone retrieval; however, this device was not required for this group of patients. With the help of the skin-kidney channel and the gravel's own gravity, the first-stage lithotripsy rate reached $98.29 \%$. Therefore, the surgical procedure adopted for this group of patients reduced the overall economic cost of treatment, benefitting both patients and doctors. Moreover, in the 2 cases where a kidney stone with a diameter of about $4 \mathrm{~mm}$ remained, 1 case was accompanied by significant hydronephrosis, and the other case had a residual stone located in the renal parenchyma at the mouth of the skinkidney channel. Both these patients were further treated by rigid percutaneous ureteroscopy through the renal channel under local anesthesia.

Even though the patients collected for this study had complicated kidney stones that were difficult to handle with a single scope, the method of single-channel minimally invasive percutaneous nephroscopy combined with retrograde soft ureteroscopy using the completely lateral and semi-lithotomy positions can also be applied to the treatment of complicated kidney stones combined with ureteral stones that are located on the same side. During the operation, ureteral stones can be washed into the kidney and then cleared out by the percutaneous renal channel, which can reduce operation time and reduce the damage caused to a patient's ureteral wall by repeated stone removal.

In summary, single-channel minimally invasive percutaneous nephroscopy combined with retrograde soft ureteroscopy in the completely lateral and semi-lithotomy positions is a safe, effective, and feasible treatment for complex kidney stones. There is no need to change a patient's position during the operation with high lithotripsy, a benefit which is highly amenable to both doctors and patients.

\section{Acknowledgments}

Funding: None.

\section{Footnote}

Reporting Checklist: The authors have completed the
STROBE reporting checklist. Available at https://dx.doi. org/10.21037/tau-21-635

Data Sharing Statement: Available at https://dx.doi. org/10.21037/tau-21-635

Conflicts of Interest: All authors have completed the ICMJE uniform disclosure form (available at https://dx.doi. org/10.21037/tau-21-635). The authors have no conflicts of interest to declare.

Ethical Statement: The authors are accountable for all aspects of the work in ensuring that questions related to the accuracy or integrity of any part of the work are appropriately investigated and resolved. All procedures performed in this study involving human participants were in accordance with the Declaration of Helsinki (as revised in 2013). The study was approved by the Ethics Committee of Peking University Shougang Hospital (No. IRBSOP-21-00) and informed consent was taken from all the patients.

Open Access Statement: This is an Open Access article distributed in accordance with the Creative Commons Attribution-NonCommercial-NoDerivs 4.0 International License (CC BY-NC-ND 4.0), which permits the noncommercial replication and distribution of the article with the strict proviso that no changes or edits are made and the original work is properly cited (including links to both the formal publication through the relevant DOI and the license). See: https://creativecommons.org/licenses/by-nc-nd/4.0/.

\section{References}

1. Matlaga BR, Jansen JP, Meckley LM, et al. Economic outcomes of treatment for ureteral and renal stones: a systematic literature review. J Urol 2012;188:449-54.

2. Ferakis N, Stavropoulos M. Mini percutaneous nephrolithotomy in the treatment of renal and upper ureteral stones: Lessons learned from a review of the literature. Urol Ann 2015;7:141-8.

3. Druskin SC, Ziemba JB. Minimally Invasive ("Mini") Percutaneous Nephrolithotomy: Classification, Indications, and Outcomes. Curr Urol Rep 2016;17:30.

4. Sakr A, Salem E, Kamel M, et al. Minimally invasive percutaneous nephrolithotomy vs standard PCNL for management of renal stones in the flank-free modified supine position: single-center experience. Urolithiasis 
2017;45:585-9.

5. Hennessey DB, Kinnear NK, Troy A, et al. Mini PCNL for renal calculi: does size matter? BJU Int 2017;119 Suppl 5:39-46.

6. Güler A, Erbin A, Ucpinar B, et al. Comparison of miniaturized percutaneous nephrolithotomy and standard percutaneous nephrolithotomy for the treatment of large kidney stones: a randomized prospective study. Urolithiasis 2019;47:289-95.

7. Proietti S, Rodríguez-Socarrás ME, Eisner B, et al. Supine percutaneous nephrolithotomy: tips and tricks. Transl Androl Urol 2019;8:S381-8.

8. Yamaguchi A, Skolarikos A, Buchholz NP, et al. Operating times and bleeding complications in percutaneous nephrolithotomy: a comparison of tract dilation methods in 5,537 patients in the Clinical Research Office of the Endourological Society Percutaneous Nephrolithotomy Global Study. J Endourol 2011;25:933-9.

9. Leng S, Xie D, Zhong Y, et al. Combined Single-Tract of Minimally Percutaneous Nephrolithotomy and Flexible Ureteroscopy for Staghorn Calculi in Oblique Supine Lithotomy Position. Surg Innov 2018;25:22-7.

10. Wang $\mathrm{Y}$, Tang $\mathrm{X}, \mathrm{Hu} \mathrm{H}$. The clinical research of 1,470 nm laser in percutaneous nephrolithotomy. Transl Androl Urol 2020;9:2172-8.

11. Feng D, Zeng X, Han P, et al. Comparison of intrarenal pelvic pressure and postoperative fever between standardand mini-tract percutaneous nephrolithotomy: a systematic review and meta-analysis of randomized controlled trials. Transl Androl Urol 2020;9:1159-66.

12. Zhu L, Jiang R, Pei L, et al. Risk factors for the fever after percutaneous nephrolithotomy: a retrospective analysis. Transl Androl Urol 2020;9:1262-9.

13. Li Y, Gu X. Lateral positioned single-channel minimal invasive percutaneous nephrolithotomy combined with retrograde flexible ureteroscope in the treatment of solitary kidney calculi: a report of 29 cases. Journal of Modern Urology 2016;21:585-8.

14. Lu GP, Liao KC, Zhang ZP, et al. The clinical study of percutaneous nephrolithotomy combined with retrograde flexible ureteroseopic lithotripsy in the prone split-leg position for treating renal staghorn calculi. Chinese Journal of Endourology (Electronic Edition) 2020;14:249-53.

15. Ping QR, Yan RP, Wang JS, et al. Percutaneous Nephrolithotomy Combined with Flexible Ureteroscopy for Complex Nephrolithiasis. Chinese Journal of Minimally Invasive Surgery 2017;17:317-21.

16. Xi JH, Zhang YB, $\mathrm{Wu} \mathrm{W}$, et al. Comparison of micro channel percutaneous nephrolithotomy combined with flexible ureteroscope holmium laser lithotripsy and dual channel percutaneous nephrolithotomy in the treatment of complex renal calculi. Journal of Clinical Urology 2019;34:820-2, 825.

17. Wang LW, Xiao RH, Xu Y, et al. Clinical observation of one stage treatment of complex renal calculi by a single channel minimally invasive percutaneous nephrolithotomy combined with flexible ureteroscope under a single position of lateral oblique lithotomy position. Journal of Clinical Urology 2017;32:422-5.

18. Zhao YL, Wang YQ, Zhang GF, et al. Clinical study on combined endoscopy in oblique supine lithotomy position in treatment of complex renal calculi. Journal of Clinical Urology 2018;33:656-8.

19. Liu YQ, Qiu M, Liu K, et al. Single-stage percutaneous nephrolithotomy combined with flexible ureteroscopy for the management of staghorn calculi with pyonephrosis. Chinese Journal of Urology 2020;41:267-71.

20. Xiao F, Chen FM, Pi SM, et al. Single-stage percutaneous nephrolithotomy combined with flexible ureteroscopy for the management of staghorn calculi with pyonephrosis. Chinese Journal of Urology 2020;35:300-3.

21. Wang YY, Jiang B, Yin XL, et al. Application of a novel fixator in percutaneous nephrolithotomy and flexible ureteroscopy combined with holmium laser lithotripsy in the treatment of complex renal calculi. Journal of Modern Urology 2020;25:625-6, 637.

22. Kim BS. Recent advancement or less invasive treatment of percutaneous nephrolithotomy. Korean J Urol 2015;56:614-23.

(English Language Editors: J. Collie and J. Gray)

Cite this article as: Pan D, Zhang L, Pan J, Yang B, Gao P, Zhang K. A retrospective analysis on the effect of singlechannel minimally invasive percutaneous nephrolithotomy combined with retrograde flexible ureteroscopy using the completely lateral decubitus and semi-lithotomy positions to treat complex kidney stones. Transl Androl Urol 2021;10(9):36383645. doi: 10.21037/tau-21-635 